\title{
MetAP2 inhibition reduces food intake and body weight in a ciliopathy mouse model of obesity
}

\author{
Tana S. Pottorf,,$^{1,2}$ Micaella P. Fagan, ${ }^{3}$ Bryan F. Burkey, ${ }^{3}$ David J. Cho, ${ }^{1,2}$ James E. Vath, ${ }^{3}$ \\ and Pamela V. Tran ${ }^{1,2}$ \\ 1Jared Grantham Kidney Institute and 'Department of Anatomy and Cell Biology, University of Kansas Medical Center \\ (KUMC), Kansas City, Kansas, USA. ${ }^{3}$ Zafgen Inc., Boston, Massachusetts, USA.
}

\begin{abstract}
The ciliopathies Bardet-Biedl syndrome and Alström syndrome are genetically inherited pleiotropic disorders with hyperphagia and obesity as primary clinical features. Methionine aminopeptidase 2 inhibitors (MetAP2i) have been shown in preclinical and clinical studies to reduce food intake, body weight, and adiposity. Here, we investigated the effects of MetAP2i administration in a mouse model of ciliopathy produced by conditional deletion of the Thm1 gene in adulthood. Thm1 conditional knockout (cko) mice showed decreased hypothalamic proopiomelanocortin expression as well as hyperphagia, obesity, metabolic disease, and hepatic steatosis. In obese Thm1-cko mice, 2-week administration of MetAP2i reduced daily food intake and reduced body weight $17.1 \%$ from baseline (vs. $5 \%$ reduction for vehicle). This was accompanied by decreased levels of blood glucose, insulin, and leptin. Further, MetAP2i reduced gonadal adipose depots and adipocyte size and improved liver morphology. This is the first report to our knowledge of MetAP2i reducing hyperphagia and body weight and ameliorating metabolic indices in a mouse model of ciliopathy. These results support further investigation of MetAP2 inhibition as a potential therapeutic strategy for ciliary-mediated forms of obesity.
\end{abstract}

Conflict of interest: MPF, BFB, and JEV were employees of Zafgen Inc., which provided funding via a sponsored research contract with KUMC

Copyright: (c) 2020, American Society for Clinical Investigation.

Submitted: October 14, 2019 Accepted: December 18, 2019 Published: December 26, 2019.

Reference information: /CI Insight. 2020;5(2):e134278.

https://doi.org/10.1172/jci. insight.134278.

\section{Introduction}

Obesity and associated insulin resistance increase risk for potentially fatal chronic diseases, including cardiovascular disease, type 2 diabetes, and nonalcoholic fatty liver disease. As obesity is pandemic and current treatments are insufficient (1), finding effective therapeutic strategies is critical to improving global health.

In preclinical and clinical studies, inhibition of methionine aminopeptidase 2 (MetAP2) has shown promising results. MetAP2 belongs to a family of metalloproteases, which cleaves the N-terminal methionine of nascent proteins. This posttranslational modification induces subcellular localization changes and activation of the targeted protein (2). Fumagillin, a natural product of Aspergillus fumigatus, irreversibly inhibits MetAP2 (3). MetAP2 inhibition causes late G1 cell cycle arrest, and inhibits cell proliferation, as well as phosphorylation of ERK1/2 (4-7). Early studies revealed anticancer and antifungal effects of fumagillin $(8,9)$. Subsequently, studies showed that fumagillin and its analogs have antiobesity effects, resulting in decreased body weight and adiposity and increased insulin sensitivity in high-fat diet-induced obese mice and rats $(10,11)$ as well as in genetic $o b / o b$ mutant mice $(12)$. Additionally, administration of the fumagillin derivative, beloranib, to individuals with nongenetic causes of obesity and to patients with Prader-Willi syndrome, a genetic disease that causes insatiable appetite and obesity, resulted in reduced food intake and body weight $(13,14)$. The effectiveness of MetAP2i in various obesity models raises the possibility that inhibiting MetAP2 may counter other forms of obesity.

Ciliopathies are genetic disorders that arise from dysfunctional or absent cilia and present numerous clinical features, including renal and hepatic fibrocystic disease, skeletal defects, infertility, hydrocephalus, mental disability, brain malformations, and central obesity (15). Primary cilia are microtubule-based, mechanosensory organelles that protrude from the apical membrane of most mammalian cells and regulate signaling pathways. Primary cilia use intraflagellar transport (IFT) multiprotein complexes for bidirectional movement of protein cargo along the ciliary axoneme. The IFT-B complex mediates anterograde protein transport, while the IFT-A complex is required for retrograde transport and for ciliary import of membrane-associated and 
A

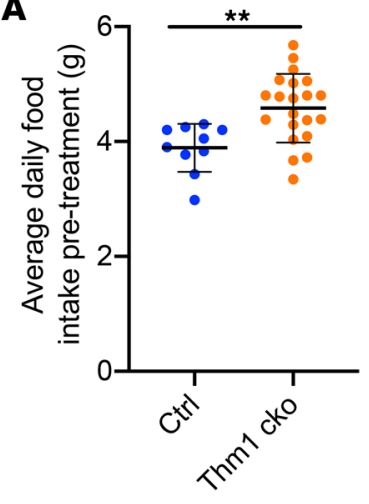

C

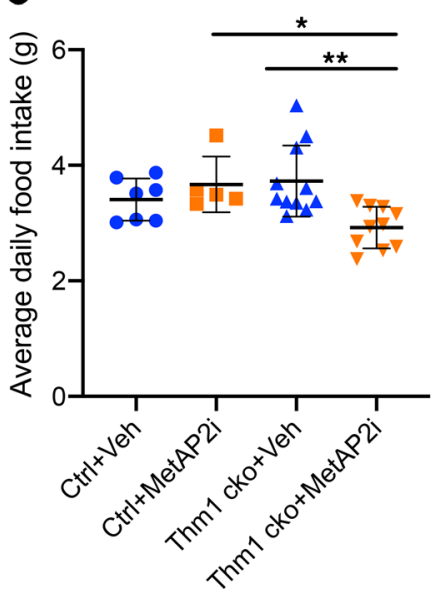

B

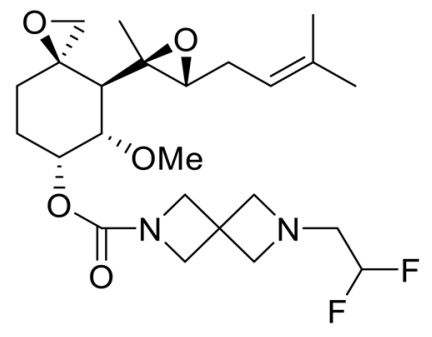

D

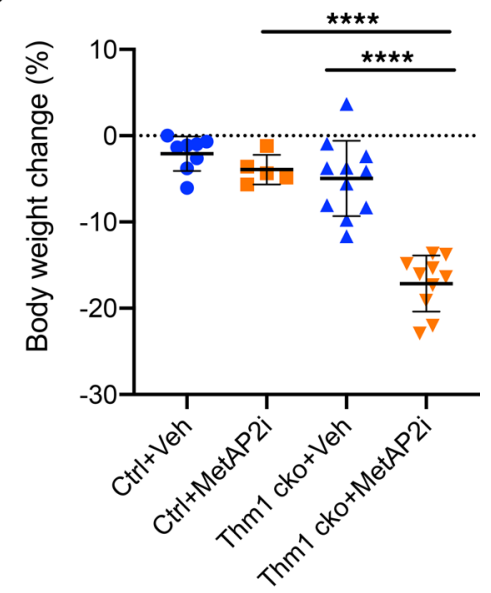

Figure 1. MetAP2i treatment decreased food intake and body weight in Thm1-cko mice. (A) Average daily food intake during week 10. (B) Chemical structure of ZCN-1258. Molecular formula: $\mathrm{C}_{24} \mathrm{H}_{36} \mathrm{~F}_{2} \mathrm{~N}_{2} \mathrm{O}_{5}$; molecular weight: $470.56 \mathrm{~g}$. (C) Average daily food intake from weeks 11 to 13. (D) Percentage of body weight change from weeks 11 to 13 . In A, statistical significance was determined by 2-tailed $t$ test. In $\mathbf{C}$ and $\mathbf{D}$, statistical significance was determined by 1-way ANOVA followed by Tukey's test. Each data point represents an individual mouse. Error bars represent mean \pm SD. ${ }^{*} P<0.05$; ${ }^{* *} P<0.005 ;{ }^{* * *} P<0.00005$.

signaling proteins $(16,17)$. Another multiprotein complex, the BBSome, transports signaling molecules to the ciliary base and acts like an adaptor between IFT complexes and protein cargo in the ciliary export of signaling molecules. Two ciliopathies, Alström syndrome and Bardet-Biedl syndrome (BBS), present obesity as a central clinical feature $(18,19)$. Additionally, polymorphisms in the $B B S$ genes in the general population have been associated with obesity, and cilia length defects have been identified in adipose-derived mesenchymal stem cells from obese individuals, suggesting a more common relevance for cilia-related mechanisms (20-22).

Modifying mutations in the IFT-A gene, THM1 (also known as TTC21B), have been reported in patients with BBS (23). We have shown that global deletion of Thm1 in adult mice causes decreased hypothalamic expression of appetite-controlling proopiomelanocortin (Pomc), hyperphagia, obesity, and metabolic syndrome (24). Here, we examined the effects of administering a potentially novel fumagillin derivative to obese Thm1 conditional knockout (cko) mice. Our results reveal reduced food intake, body weight, and adipose tissue mass as well as improved metabolic indices. These data indicate MetAP2 inhibition as a potential therapeutic strategy against obesity caused by genetic disorders of cilia.

\section{Results}

MetAP2i treatment decreases body weight, food intake, and adiposity in Thm1-cko mice. To generate obese Thm1-cko mice, we induced deletion of Thm1 in male mice at 5 weeks of age and fed mutant mice and control littermates ad libitum throughout the 13-week study (Supplemental Figure 1A; supplemental material available online with this article; https://doi.org/10.1172/jci.insight.134278DS1). 
A

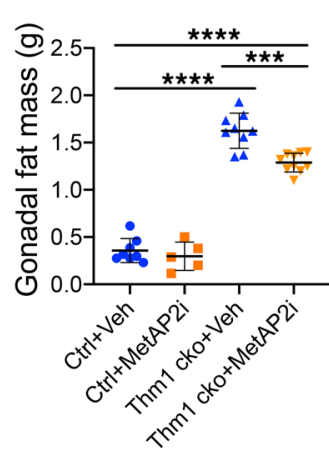

E

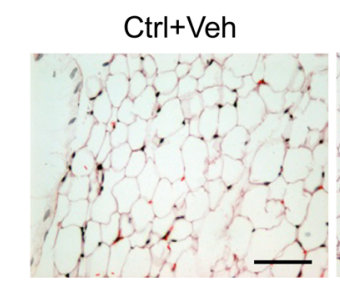

$\mathbf{F}$

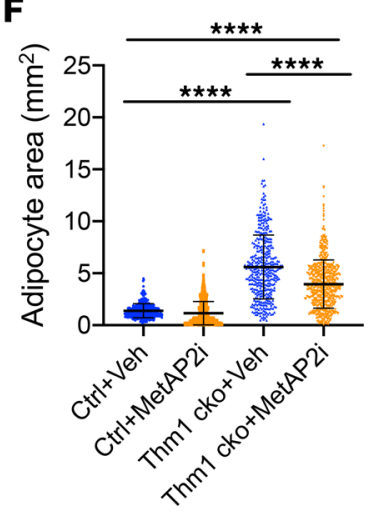

B

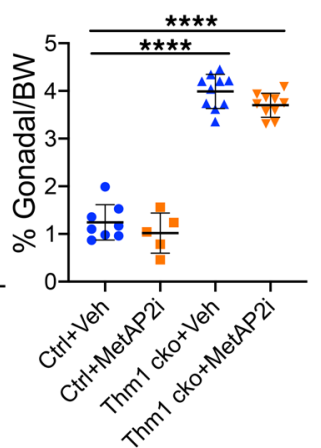

C

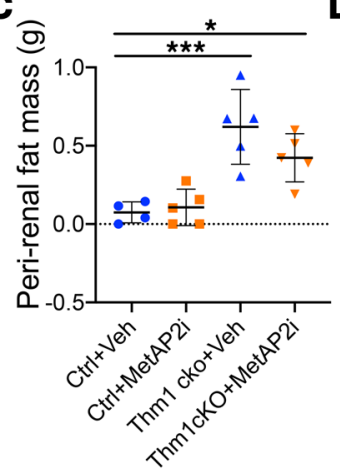

D

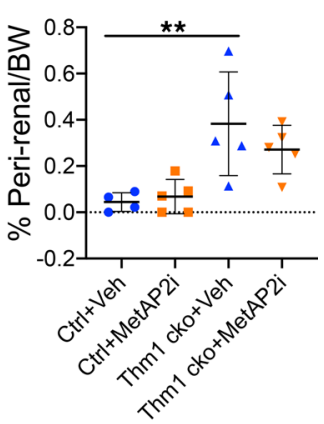

Thm1 cko+Veh

Thm1 cko+MetAP2i

Ctrl+MetAP2i
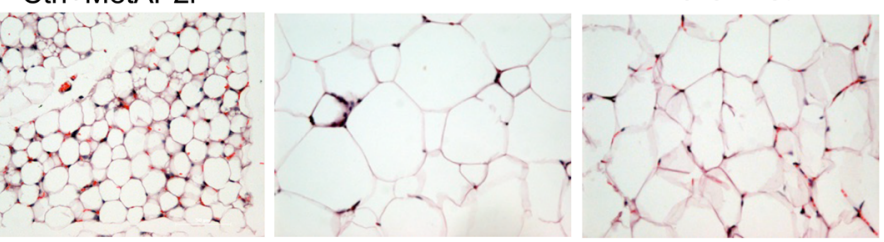

Figure 2. MetAP2i treatment decreased gonadal fat mass and adipocyte size in Thm1-cko mice. (A) Gonadal adipose mass. (B) Percentage of gonadal adipose mass/body weight. (C) Perirenal adipose mass. (D) Percentage of perirenal adipose mass/body weight. Each data point represents an individual mouse. (E) H\&E staining of gonadal adipose tissue. Scale bar: $50 \mu \mathrm{m}$. (F) Quantification of gonadal adipocyte size from $n=2$ control mice/group and $n=3$ Thm1-cko mice/group. Each data point represents an adipocyte. Error bars represent mean $\pm \mathrm{SD}$. Statistical significance was determined by 1-way ANOVA followed by Tukey's test. ${ }^{*} P<0.05$; ${ }^{* *} P<0.005 ;{ }^{* * *} P<0.0005$; ${ }^{* * * *} P<0.00005$.

Body weight was measured weekly from 0 to 10 weeks after Thm1 deletion. At 10 weeks following gene deletion, Thm1-cko mice and control littermates weighed $42.2 \pm 1.1 \mathrm{~g}$ and $29.4 \pm 0.7 \mathrm{~g}$, respectively, confirming the obese phenotype of the mutant mice (Supplemental Figure 1B). At this time point (week 10 of the experiment), mice were housed individually and baseline measurements of food intake and body weight were obtained daily for 1 week. Thm1-cko mice showed an approximately $15 \%$ higher average daily food intake than control littermates, consistent with hyperphagia (Figure 1A). Following 1 week of solitary housing, Thm1-cko mice and control littermates weighed $41.17 \pm 1.1 \mathrm{~g}$ and $29.18 \pm 0.7$ g, respectively (Supplemental Figure 1, C and D). We next administered daily subcutaneous injections of a MetAP2 inhibitor (MetAP2i), ZGN-1258 (Figure 1B), or vehicle for 2 weeks (weeks 11-13). In Thm1-cko mice, MetAP2i treatment reduced food intake relative to vehicle (Figure 1C). Additionally, in Thm1-cko mice, MetAP2i treatment caused a $-17.1 \%$ body weight reduction compared with $-5.0 \%$ for vehicle (Figure 1D and Supplemental Figure 1E). These data show that MetAP2i counters the hyperphagia and increased body weight induced by deletion of Thm1.

We next analyzed gonadal and perirenal fat depots, which we have shown previously to be increased in obese Thm1-cko mice (24). As expected, vehicle-treated Thm1-cko mice showed increased gonadal and renal adipose tissue mass relative to control littermates (Figure 2, A-D). In Thm1-cko mice, MetAP2i treatment reduced gonadal fat mass compared with vehicle treatment (Figure 2A). Histology of gonadal fat pads showed an increase in gonadal adipocyte cell size in vehicle-treated Thm1-cko mice relative to control mice (Figure 2, E and F), consistent with previous findings (24). However, gonadal adipocyte cell size was reduced in MetAP2i-treated mutants. These data show that MetAP2i treatment partially attenuates the increased gonadal adipose tissue mass and adipocyte size caused by deletion of Thm1. 
A

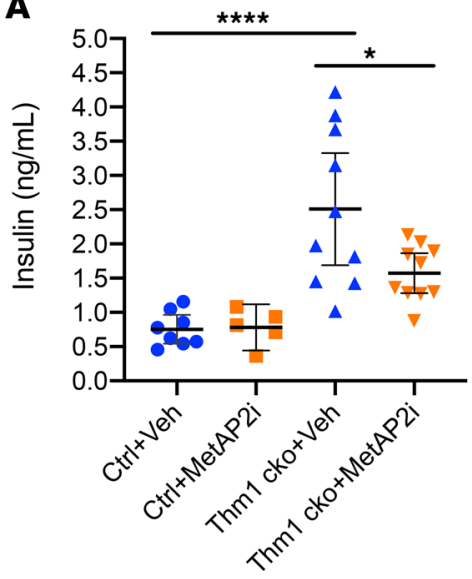

B

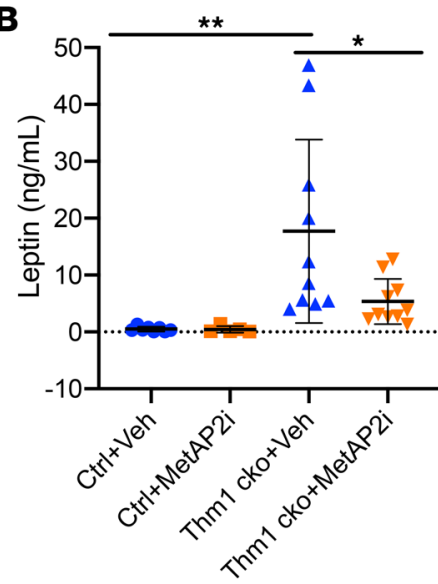

Figure 3. MetAP2i treatment improved metabolic parameters in Thm1-cko mice. Nonfasting levels of serum (A) insulin and (B) leptin. Each data point represents an individual mouse. Error bars represent mean \pm SD. Statistical significance was determined by 1-way ANOVA followed by Tukey's test. ${ }^{*} P<0.05 ;{ }^{* *} P<0.005 ;{ }^{* * *} P<0.00005$.

MetAP2i treatment corrects the hyperinsulinemia, hyperleptinemia, and hepatosteatosis in Thm1-cko mice. Following the 2-week intervention, we measured metabolic parameters, including nonfasting blood glucose, serum insulin, and leptin. In Thm1-cko mice, MetAP2i treatment reduced nonfasting blood glucose levels relative to those after vehicle treatment (Supplemental Figure 2). As we observed previously (24), serum insulin levels were higher in vehicle-treated Thm1-cko mice than in vehicle-treated control littermates, but MetAP21 treatment in Thm1-cko mice decreased insulin levels to such an extent that levels were not significantly different from those of vehicle-treated control mice (Figure 3A). Similarly, serum leptin was elevated in vehicle-treated Thm1-cko mice relative to control littermates, but MetAP2i treatment in Thm1-cko mice reduced leptin levels to the degree that leptin levels were not significantly different from those of vehicle-treated control mice (Figure 3B). These data indicate that MetAP2i can correct these metabolic parameters.

Obese Thm1-cko mice also develop hepatic steatosis (24). To determine whether MetAP2i treatment affected the liver, we examined the histology of livers of vehicle- and MetAP2i-treated mice. Vehicle-treated Thm1-cko mice had vacuoles in their livers, suggesting formation of lipid droplets (Figure 4 and Supplemental Figure 3). These vacuoles were not observed in control mice. Further, the vacuoles were reduced or absent in livers of MetAP2i-treated Thm1-cko mice, suggesting that the improved metabolism resulting from MetAP2i treatment extends to the liver.

Since many drugs are metabolized and excreted by the kidney, we examined the effects of MetAP2i in the kidney. Vehicle-treated Thm1-cko mice had similar kidney weights to those of control mice (Supplemental Figure 4A) but showed a slight decrease in the kidney weight/body weight ratio (Supplemental Figure 4B). This is likely due to the increased body weight of Thm1-cko mice, since the kidney weight/ body weight ratio of MetAP2i-treated Thm1-cko mice was not significantly lower than that of control mice. Importantly, MetAP2i treatment of control or Thm1-cko mice did not affect kidney morphology relative to vehicle (Supplemental Figure 4C), suggesting that the MetAP2i treatment did not have nephrotoxic effects. We next examined primary cilia by immunostaining for the ciliary membrane protein, ARL13B, together with incubating with the lectin, Dolichos biflorus agglutinin, to label the collecting duct (Supplemental Figure 4D). We observed previously that cilia are shortened in Thm1-deficient mice (24-26). Average cilia length in collecting ducts of vehicle-treated Thm1-cko mice was shorter than that of vehicle-treated control mice, but we noted a wide range of cilia lengths in the Thm1-cko collecting ducts and statistical significance was not achieved (Supplemental Figure 4E). Unexpectedly, we observed that cilia lengths in collecting ducts of MetAP2i-treated mice were longer than in vehicle-treated mice.

\section{Discussion}

In this study, we provide the first evidence to our knowledge that MetAP2 inhibition reduces food intake and body weight and substantially improves metabolic parameters in a ciliary model of obesity. These data broaden the therapeutic applicability of MetAP2 inhibition and demonstrate an effective pharmacological intervention in a ciliopathy rodent model of obesity. 

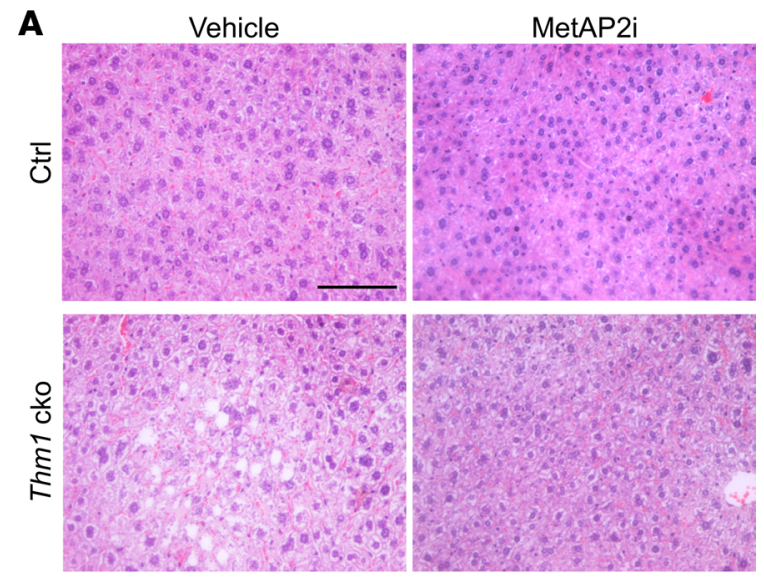

$\mathbf{B}$

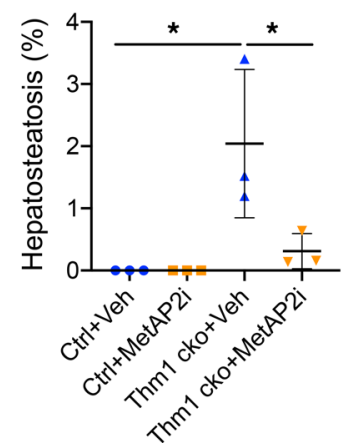

Figure 4. MetAP2i treatment improved liver morphology in Thm1-cko mice. (A) Liver histology of vehicle- and MetAP2i-treated control and Thm1-cko littermates. Scale bar: $100 \mu \mathrm{m}$. Livers of mice from 2 additional litters are shown in Supplemental Figure 3. Patches of circular white spaces were observed in vehicle-treated Thm1-cko mice and not in MetAP2i-treated Thm1-cko mice. (B) Quantification of hepatic steatosis/image from $n=3$ mice/group. Fields including patches of circular white spaces were imaged, and entire fields were quantified. Error bars represent mean \pm SD. Statistical significance was determined by 1-way ANOVA followed by Tukey's test. ${ }^{*} P<0.05$.

Previously we showed that Thm1 deletion in adult mice results in decreased hypothalamic expression of the appetite-controlling neuropeptide, Pomc, before the mutant mice gain significant body weight, suggesting reduced Pomc may drive the obese phenotype (24). Consistent with this notion, Pomc-null mice are obese (27). Additionally, in mice fed a high-fat diet, reduced hypothalamic Pomc expression was found to be the earliest marker predicting obesity (28). The ability of MetAP2 inhibition to be effective in various models suggests that MetAP2 acts on a common pathway that is misregulated in all models, leading to increased appetite and obesity. Since MetAP2i reduced food intake in Thm1-cko mice, MetAP2i may have targets in neuronal cells either downstream of or countering the effects of reduced Pomc expression.

Recently, we have also found that hyperphagia in Thm1-cko mice induces hepatic insulin resistance, which, in turn, drives systemic insulin resistance prior to an obese phenotype (29). Thus, the corrected metabolic indices in MetAP2i-treated Thm1-cko mice may also be due in part to the reduced food intake.

MetAP2i treatment also resulted in decreased adipocyte cell size, consistent with previous studies in rodent models of high-fat diet-induced obesity $(10,11)$. Smaller, metabolically healthier adipocytes are associated with increased adipogenesis, yet whether MetAP2i directly affects adipogenesis is still unclear. In one study, addition of fumagillin to an in vitro preadipocyte differentiation assay promoted adipogenesis but had minimal effects in an in vivo adipogenesis assay (30). In contrast, another study showed that a fumagillin derivative inhibited adipogenesis in an in vitro preadipocyte differentiation study, but fumagillin treatment of cells enhanced glucose uptake, indicative of metabolically healthier cells (31). Alternatively, a prevailing hypothesis is that the antiangiogenic effects of fumagillin reduce adipose tissue mass, yet angiogenesis changes did not drive reduction of adipose tissue mass in mice (10). Thus, further studies are required to determine the mechanisms by which adipocyte size and adipose tissue mass are reduced by MetAP2i. 
Interestingly, MetAP2i treatment did not affect control mice. This might be because the effectiveness of MetAP2 inhibitors depends on MetAP activity within a cell. Indeed, MetAP2 expression is either upregulated or has altered localization in tissues of obese mice (32). Further, a cancer study has demonstrated that a cell's response to fumagillin depends on the expression of both MetAP1 and MetAP2, revealing that MetAP2i treatment affects cell types differentially (33). In this light, querying the expression of MetAP1 and MetAP2 in various cell types, such as brain, liver, fat, and endothelial cells, in obese and lean mice may be beneficial in determining the cells that will be most responsive to MetAP2i treatment.

Unexpectedly, we observed during the first week of individual housing prior to treatment that many of the control mice and all of the Thm1-cko mice showed reduced weight loss. Moreover, the mutant animals showed a greater percentage of weight reduction $(-3.8 \%)$ than control animals $(-0.8 \%$; Supplemental Figure 1, B-D). This observation indicates that caution is required when analyzing data that involve mice that are individually housed, which can generate stress and confound results. Importantly, MetAP2i treatment resulted in greater weight reduction than single housing alone.

Due to safety issues, Zafgen Inc. has recently discontinued the development of MetAP2 inhibitors (34). In patients with Prader-Willi Syndrome, the MetAP2 inhibitor, beloranib, was successful in lowering food intake and body weight, but it also caused an adverse event, venous thromboembolism (14). Prolonged exposure to MetAP2i by endothelial cells causes cell cycle arrest (35) and may initiate cell signaling pathways that promote coagulation (36). Thus, next-generation MetAP2 inhibitors that are designed to have minimal exposure to endothelial cells may provide a solution (36). Alternatively, reversible MetAP2 inhibitors may also reduce these adverse events (37). A reversible MetAP2 inhibitor, M8891, is currently in a phase I clinical trial for cancer (38).

Our study indicates that there are plausible targets of MetAP2 inhibition that effectively reduce hyperphagia and body weight and substantially improve metabolic parameters in a ciliopathy model. This substantiates the need for greater understanding of the biology of MetAP2 (39). Currently, agonists of the melanocortin 4 receptor, which is activated by the processed protein products of Pomc, are being tested in clinical trial to target obesity in patients with BBS and Alström syndrome (40). Aside from this, a potential therapy for ciliopathy-induced obesity has not been demonstrated. The mechanism by which MetAP2 inhibition exerts its antiobesity effects remains elusive. Intriguingly, our data suggest a potential connection between MetAP2 and cilia biology. While these observations were made in the kidney and are not directly relevant to the attenuated obesity and metabolic defects in MetAP2i-treated Thm1-cko mice, future investigations between MetAP2 inhibition and ciliary dynamics in metabolically relevant cells may reveal potential mechanisms and therapeutic targets.

\section{Methods}

Generation of Thm1-cko mice. Thm1-cko mice were maintained on a mixed FVB/C57BL/6J background and generated as described previously (24), using a Thm1-null allele (called aln), a Thm1-floxed allele that has LoxP sites flanking exon 4, and a tamoxifen-inducible ROSA26-Cre ${ }^{E R T}$ recombinase (Jackson Laboratories, stock 004847), which is expressed globally. Thm $1^{f / f l}$ females were mated to Thm $1^{a \ln /+} ; R O S A 26-C r e^{E R T}$ males to produce Thm1 $1^{f l a l n}$; ROSA26-Cre ${ }^{E R T}$ (Thm1-cko mice) and Thm1 $1^{f /+} ;$ ROSA26-Cre ${ }^{E R T}$ (control mice). Mice were genotyped via PCR using the following primers: (a) alndiag-F 5'-CGCTGATTAACTACTATGGTC-3'; (b) alndiag-R 5'-GCGTGGTAAAATCGGAAGAC-3'; (c) Thm1 ${ }^{\mathrm{fl}}$-F 5'-AAGTGTTTGAAAACCTGAATGGA-3'; (d) Thm1 fl-R 5'-GCACAGACTCCTGCTTCTCA-3'; (e) Cre-F 5'-GCGGTCTGGCAGTAAAAACTATC-3'; and (f) Cre-R 5'-GTGAAACAGCATTGCTGTCACTT-3'. The aln PCR product was digested with AvaII, which cleaves the aln allele into 90-bp and 19-bp products. Cre recombinase expression was induced at 5 weeks of age by i.p. injection of $10 \mathrm{mg}$ tamoxifen/40 g mouse weight. Both control and Thm1-cko mice received tamoxifen (MilliporeSigma, T5648), which was suspended in corn oil (MilliporeSigma, C8267) at $30 \mathrm{mg} / \mathrm{ml}$. Only male mice were used and were maintained on a regular laboratory rodent chow diet (PicoLab, 5053).

Administration of MetAP2i. Mice were fed ad libitum throughout the duration of the study. Body weight was measured weekly from 0 to 10 weeks after tamoxifen injection to ascertain the obese phenotype in Thm1cko mice. Beginning at 10 weeks after tamoxifen injection, mice were housed individually, and food intake and body weight were measured daily until the end of the experiment. Subcutaneous injections of MetAP2i (ZGN-1258, $0.3 \mathrm{mg} / \mathrm{kg} / \mathrm{d})$ or vehicle ( $5 \%$ mannitol) were administered daily from 11 to 13 weeks after tamoxifen injection. This dose of ZGN-1258 reduced food intake and body weight in $o b / o b$ mice, and these effects correlated with target engagement, as measured by levels of the MetAP2-specific substrate, thioredoxin with intact N-terminal methionine (THX1-6), in brain and peripheral tissues (our unpublished observations). 
Blood/serum and organ retrieval. At the end of the study, mice were weighed using a laboratory digital measuring scale (Maxx series MXX-123; Denver Instrument). Tails were nicked to obtain tail blood to measure nonfasting blood glucose using Bayer Blood Glucose Contour Strips together with the Bayer Contour Blood Glucose Meter system. Following euthanization of mice, organs, including gonadal and perirenal fat pads and kidneys, were dissected and weighed. Tissues were submerged in $10 \%$ formalin at $4^{\circ} \mathrm{C}$ for $3-7$ days. Trunk blood was collected in a Microvette CB300z blood collection tube (Kent Scientific), and serum was isolated by centrifuging blood collection tubes for 6 minutes at $800 \mathrm{~g}$ at $4^{\circ} \mathrm{C}$ using a tabletop centrifuge (PrismR, C2500-R). Serum was stored at $-80^{\circ} \mathrm{C}$ until measurements of insulin and leptin were performed.

Histology. Following fixation in $10 \%$ formalin at $4^{\circ} \mathrm{C}$ for 3-7 days, tissues were dehydrated through an ethanol series, paraffin embedded, and sectioned at $7-\mu \mathrm{m}$ thicknesses. Sections were rehydrated and stained with hematoxylin and eosin using a standard protocol. Staining was viewed and imaged using a Nikon 80 microscope equipped with a Nikon DS-Fi1 camera.

Serum insulin and leptin measurements. Serum insulin and leptin levels were measured using Mouse Ultrasensitive Insulin ELISA and Mouse/Rat Leptin ELISA kits (ALPCO) according to manufacturer's instructions.

Immunofluorescence. Immunofluorescence was performed as described previously (41). Sections were rehydrated. Heat antigen retrieval was performed in trisodium citrate solution, $\mathrm{pH}$ 6.0, using a steamer. Sections were rinsed in distilled water (10 times) and then blocked in 2\% BSA in PBS for 1 hour. Arl13B antibody (Proteintech) was diluted in blocking buffer (2\% BSA in PBS), and tissue sections were incubated with primary antibody at $4^{\circ} \mathrm{C}$ overnight. Sections were washed 3 times in PBS and then incubated with Dolichos biflorus agglutinin (Vector Laboratories) for 1 hour at room temperature. Sections were washed in PBS 3 times and then incubated with Alexa Fluor 594 goat anti-rabbit secondary antibody (Thermo Fisher) for 1 hour at room temperature. Following 3 washes in PBS, tissue sections were then mounted with Fluoromount G containing DAPI mounting media (Electron Microscopy Services). Immunolabeled tissues were viewed and imaged using a Leica TCS SPE confocal microscope configured on a DM550 Q upright microscope.

Statistics. Graphpad Prism 8 was used to perform statistical analyses, which included 2-tailed $t$ tests and 1-way ANOVA followed by Tukey's test. $P$ values of less than 0.05 were considered significant.

Study approval. All animal studies were approved by the Institutional Animal Care and Use Committee of KUMC.

\section{Author contributions}

MPF, BFB, JEV, and PVT designed the research. TSP, DJC, and PVT performed the experiments. TSP, MPF, BFB, DJC, JEV, and PVT analyzed the data. TSP and PVT wrote the paper.

\section{Acknowledgments}

We thank members of the KUMC Department of Anatomy and Cell Biology and Kidney Institute for helpful discussions. We also thank the KUMC Kansas Intellectual and Developmental Disabilities Research Center Core Services, Jing Huang of the Histology Core, and Michelle Winter of the Animal Behavioral Core. Work performed by the cores is supported by the KUMC Smith Intellectual and Developmental Disabilities Research Center (NIH U54 HD 090216). PVT is supported by R01 DK103033.

Address correspondence to: Pamela V. Tran, University of Kansas Medical Center, 3901 Rainbow Boulevard, MS \#3038, Kansas City, Kansas 66160, USA. Phone: 913.945.7325; Email: ptran@kumc.edu.

1. Roth J, Qiang X, Marbán SL, Redelt H, Lowell BC. The obesity pandemic: where have we been and where are we going? Obes Res. 2004;12 Suppl 2:88S-101S.

2. Lowther WT, Matthews BW. Structure and function of the methionine aminopeptidases. Biochim Biophys Acta. 2000;1477(12):157-167

3. Liu S, Widom J, Kemp CW, Crews CM, Clardy J. Structure of human methionine aminopeptidase-2 complexed with fumagillin. Science. 1998;282(5392):1324-1327.

4. Turk BE, Griffith EC, Wolf S, Biemann K, Chang YH, Liu JO. Selective inhibition of amino-terminal methionine processing by TNP-470 and ovalicin in endothelial cells. Chem Biol. 1999;6(11):823-833.

5. Yeh JR, Mohan R, Crews CM. The antiangiogenic agent TNP-470 requires p53 and p21CIP/WAF for endothelial cell growth arrest. Proc Natl Acad Sci USA. 2000;97(23):12782-12787.

6. Datta B, Datta R, Majumdar A, Ghosh A. The stability of eukaryotic initiation factor 2-associated glycoprotein, p67, increases during skeletal muscle differentiation and that inhibits the phosphorylation of extracellular signal-regulated kinases 1 and 2 . Exp Cell Res. 2005;303(1):174-182. 
7. Shimizu H, Yamagishi S, Chiba H, Ghazizadeh M. Methionine aminopeptidase 2 as a potential therapeutic target for human non-small-cell lung cancers. Adv Clin Exp Med. 2016;25(1):117-128.

8. Dipaolo JA, Tarbell DS, Moore GE. Studies on the carcinolytic activity of fumagillin and some of its derivatives. Antibiot Annu. 1958;6:541-546.

9. Garvey MJ, Ambrose PG, Ulmer JL. Topical fumagillin in the treatment of microsporidial keratoconjunctivitis in AIDS. Ann Pharmacother. 1995;29(9):872-874.

10. Lijnen HR, Frederix L, Van Hoef B. Fumagillin reduces adipose tissue formation in murine models of nutritionally induced obesity. Obesity (Silver Spring). 2010;18(12):2241-2246.

11. An J, et al. Physiological mechanisms of sustained fumagillin-induced weight loss. JCI Insight. 2018;3(5):99453.

12. Bråkenhielm E, et al. Angiogenesis inhibitor, TNP-470, prevents diet-induced and genetic obesity in mice. Circ Res. 2004;94(12):1579-1588.

13. Hughes TE, Kim DD, Marjason J, Proietto J, Whitehead JP, Vath JE. Ascending dose-controlled trial of beloranib, a novel obesity treatment for safety, tolerability, and weight loss in obese women. Obesity (Silver Spring). 2013;21(9):1782-1788.

14. McCandless SE, et al. Effects of MetAP2 inhibition on hyperphagia and body weight in Prader-Willi syndrome: A randomized, double-blind, placebo-controlled trial. Diabetes Obes Metab. 2017;19(12):1751-1761.

15. Baker K, Beales PL. Making sense of cilia in disease: the human ciliopathies. Am J Med Genet C Semin Med Genet. 2009;151C(4):281-295.

16. Mukhopadhyay S, et al. TULP3 bridges the IFT-A complex and membrane phosphoinositides to promote trafficking of G protein-coupled receptors into primary cilia. Genes Dev. 2010;24(19):2180-2193.

17. Fu W, Wang L, Kim S, Li J, Dynlacht BD. Role for the IFT-A complex in selective transport to the primary cilium. Cell Rep. 2016;17(6):1505-1517.

18. Guo DF, Rahmouni K. Molecular basis of the obesity associated with Bardet-Biedl syndrome. Trends Endocrinol Metab. 2011;22(7):286-293

19. Girard D, Petrovsky N. Alström syndrome: insights into the pathogenesis of metabolic disorders. Nat Rev Endocrinol. 2011;7(2):77-88.

20. Benzinou M, et al. Bardet-Biedl syndrome gene variants are associated with both childhood and adult common obesity in French Caucasians. Diabetes. 2006;55(10):2876-2882.

21. Ritter A, et al. Primary cilia are dysfunctional in obese adipose-derived mesenchymal stem cells. Stem Cell Reports. 2018;10(2):583-599

22. Ritter A, Louwen F, Yuan J. Deficient primary cilia in obese adipose-derived mesenchymal stem cells: obesity, a secondary ciliopathy? Obes Rev. 2018;19(10):1317-1328.

23. Davis EE, et al. TTC21B contributes both causal and modifying alleles across the ciliopathy spectrum. Nat Genet. 2011;43(3):189-196

24. Jacobs DT, et al. Dysfunction of intraflagellar transport-A causes hyperphagia-induced obesity and metabolic syndrome. Dis Model Mech. 2016;9(7):789-798.

25. Tran PV, et al. THM1 negatively modulates mouse sonic hedgehog signal transduction and affects retrograde intraflagellar transport in cilia. Nat Genet. 2008;40(4):403-410.

26. Tran PV, et al. Downregulating hedgehog signaling reduces renal cystogenic potential of mouse models. J Am Soc Nephrol. 2014;25(10):2201-2212.

27. Yaswen L, Diehl N, Brennan MB, Hochgeschwender U. Obesity in the mouse model of pro-opiomelanocortin deficiency responds to peripheral melanocortin. Nat Med. 1999;5(9):1066-1070.

28. Souza GF, et al. Defective regulation of POMC precedes hypothalamic inflammation in diet-induced obesity. Sci Rep. 2016;6:29290

29. Jacobs DT AB, et al. Intraflagellar-transport A dysfunction causes hyperphagia-induced systemic insulin resistance in a preobese state [published online ahead of print November 19, 2019]. FASEB J. doi: 10.1096/fj.201900751R.

30. Scroyen I, Christiaens V, Lijnen HR. Effect of fumagillin on adipocyte differentiation and adipogenesis. Biochim Biophys Acta. 2010;1800(4):425-429.

31. Siddik MAB, Das BC, Weiss L, Dhurandhar NV, Hegde V. A MetAP2 inhibitor blocks adipogenesis, yet improves glucose uptake in cells. Adipocyte. 2019;8(1):240-253.

32. Han J, Tang Y, Lu M, Hua H. Comprehensive comparison of MetAP2 tissue and cellular expression pattern in lean and obese rodents. Diabetes Metab Syndr Obes. 2018;11:565-577.

33. Frottin F, et al. MetAP1 and MetAP2 drive cell selectivity for a potent anti-cancer agent in synergy, by controlling glutathione redox state. Oncotarget. 2016;7(39):63306-63323.

34. Zafgen. Zafgen to explore strategic alternatives. http://zafgen.gcs-web.com/news-releases/news-release-details/zafgenexplore-strategic-alternatives. Updated September 5, 2019. Accessed December 26, 2019.

35. Zhang Y, Griffith EC, Sage J, Jacks T, Liu JO. Cell cycle inhibition by the anti-angiogenic agent TNP-470 is mediated by p53 and p21WAF1/CIP1. Proc Natl Acad Sci USA. 2000;97(12):6427-6432.

36. Burkey BF, Hoglen NC, Inskeep P, Wyman M, Hughes TE, Vath JE. Preclinical efficacy and safety of the novel antidiabetic, antiobesity metap2 inhibitor ZGN-1061. J Pharmacol Exp Ther. 2018;365(2):301-313.

37. Heinrich T, et al. Discovery and structure-based optimization of next-generation reversible methionine aminopeptidase-2 (MetAP-2) inhibitors. J Med Chem. 2019;62(10):5025-5039.

38. Heinrich T, et al. Identification of methionine aminopeptidase-2 (MetAP-2) inhibitor M8891: A clinical compound for the treatment of cancer. J Med Chem. 2019;62(24):11119-11134.

39. Chang YH. Common therapeutic target for both cancer and obesity. World J Biol Chem. 2017;8(2):102-107.

40. Eneli I, et al. Tracing the effect of the melanocortin-4 receptor pathway in obesity: study design and methodology of the TEMPO registry. Appl Clin Genet. 2019;12:87-93.

41. Silva LM, Wang W, Allard BA, Pottorf TS, Jacobs DT, Tran PV. Analysis of primary cilia in renal tissue and cells. Methods Cell Biol. 2019;153:205-229. 